\title{
4 \\ APROXIMACIÓN A LAS REPRESENTACIONES Y CREENCIAS DEL ALUMNADO DE MAGISTERIO SOBRE LOS ESTILOS DE ENSEÑANZA
}

\section{(APPROACH TO REPRESENTATIONS AND BELIEFS OF STUDENTS OF THE TEACHER TRAINING DEGREE IN RELATION TO TEACHING STYLES)}

Margarita González-Peiteado

Universidad Nacional de Educación a Distancia

Margarita Pino-Juste

Universidad de Vigo

DOI: 10.5944/educxx1.17.1.10706

\section{Cómo referenciar este artículo/How to reference this article:}

González Peiteado, M. y Pino Juste, M. (2014). Aproximación a las representaciones y creencias del alumnado de Magisterio sobre los estilos de enseñanza. Educación XX1, 17 (1), 83-110. doi: 10.5944/educxx1.17.1.10706.

González Peiteado, M. y Pino Juste, M. (2014). Approach to representations and beliefs of students of the teacher training degree about teaching styles. Educación XX1, 17 (1), 83-110. doi: 10.5944/educxx1.17.1.10706.

\section{RESUMEN}

El artículo presenta los resultados de una investigación desarrollada en las tres Universidades de Galicia con el propósito de descubrir los estilos de enseñanza preferidos por el alumnado de las distintas especialidades de Magisterio.

Se trata de un diseño de investigación no experimental de carácter transversal. Se utilizó la triangulación como recurso de investigación utilizando como instrumentos los grupos de discusión, la entrevista y una escala Likert.

Entre los resultados destaca que el estilo activo es el preferido por los estudiantes frente al estilo académico. Además hemos de señalar que los hombres en comparación con las mujeres, manifiestan una mayor tendencia hacia estilos tradicionales.

\section{ABSTRACT}

This paper presents the results of research conducted at the three Universities of Galicia in order to discover the teaching styles preferred by students pertaining to the Teacher Training Degree. 
This is a non-experimental crosscurricular research design. Triangulation was used as a research method with instruments like focus groups, interviews and a Likert scale.

The results show that the active style is preferred by students opposite to the traditional academic style. We have also noted that men compared to women, show a greater tendency towards traditional styles.

\section{MARCO TEÓRICO}

Los cambios generalizados y acelerados que diseñan el panorama actual en todos los ámbitos de la actividad humana han incidido notoriamente sobre el rol a desempeñar por el docente. Tras un largo recorrido trazado por distintos períodos históricos y concepciones pedagógicas, se perfila un nuevo escenario educativo en el que los cambios formativos no solo apuntan a las competencias técnicas y científicas, sino también a las competencias sociales necesarias para asumir nuevos retos, lo que les ha permitido a los futuros profesores el desarrollo de perfiles cada vez más profesionales. Desde este modelo, se considera que la eficacia profesional está vinculada con la calidad del trabajo que realizan, el compromiso que asumen en su trayectoria laboral, así como con el estilo de enseñanza que implementan en las aulas, entre otros aspectos.

Esta evolución en la percepción de la praxis educativa ha implicado necesariamente un rechazo de estilos tradicionales y un compromiso de coherencia con las demandas que plantea la sociedad, estableciendo estilos de enseñanza abiertos, innovadores, flexibles y comprensivos, venciendo la resistencia al cambio. Dimensiones, todas ellas, que deben ser atendidas desde la Formación Inicial del Profesorado (Blanco Felip, Jové Deltell y Reverter Masià, 2012; Feiman-Nemser, 2001; Marcelo, 2009).

Obviamente, lo asimilado a lo largo de la carrera no garantiza que los futuros docentes lo vayan a aplicar en las aulas; pero todos sabemos que lo que no se aprende, no se puede enseñar. El hecho de aproximarnos a distintos enfoques de la enseñanza nos ofrece la posibilidad de adaptarnos a las diversas necesidades e intereses del alumnado y abre la reflexión sobre la conveniencia de implantar un estilo u otro de enseñanza adaptándose a las circunstancias. Pero, ¿qué entendemos por estilo de enseñanza?

Considerando que las diferencias individuales son un fenómeno intrínseco y concomitante al desarrollo y evolución del ser humano, podemos afirmar que la realidad particular del ejercicio docente presenta como nota fundamental la variedad de constructos cognitivos, culturales y con- 
textuales. Todo ello como resultado de una historia personal, académica y profesional que va configurando consciente o inconscientemente un estilo preferido de dirigir la acción educativa. «La docencia es una creación científica y artística y por tanto, muy personal» (Nevot y Cuevas, 2009, p. 40).

Es desde la aceptación de esta diversidad como puede comprenderse que cada persona presente una determinada forma de enseñar y de aprender, manifestando una variedad de conductas y roles a lo largo del proceso educativo, contribuyendo a la conformación de un estilo determinado. Así lo suscribe Lozano (2004, p. 17) al proporcionarnos a partir de sus reflexiones un conjunto de rasgos que creemos importantes para poder establecer un perfil docente. "Si bien es cierto que un estilo implica preferencias, tendencias y disposiciones, también lo es el hecho de que existan patrones conductuales y fortalezas que distinguen a un sujeto de los demás en la manera en que se conduce, se viste, habla, piensa, aprende y enseña».

En cuanto a este aspecto, en el trabajo de Gonzaléz Peiteado (2010, p. 3) se resalta que en el comportamiento docente existe una implicación de multiplicidad de factores relacionados con una ideología o percepción de la realidad que asume el educador. Así, para esta autora, "Cuando se habla de estilos de enseñanza se alude al modo particular de enseñar, a la tendencia docente de adoptar un determinado modo de interactuar con el alumno en función de las demandas específicas de la tarea, de percibir las necesidades del educando, sus intereses, aptitudes, actitudes, de pensar acerca de la praxis educativa, pero también se refiere a comportamientos verbales y no verbales, producto de creencias, principios, autobiografía y teorías de la enseñanza que sustentan su actuación refrendado por una cierta regularidad en la forma de aplicar las distintas estrategias de enseñanza».

En efecto, los estilos de enseñanza vertebran dimensiones que tienen gran impacto en el ejercicio de la profesión, en la configuración pedagógica del quehacer educativo y en las relaciones interpersonales que se establecen dentro del aula (González-Peiteado, 2012). Se trata de una dimensión que constituye una articulación entre lo individual y lo social. Por tanto, entendemos que ambas dimensiones van asociados a características cognitivas, afectivas y procedimentales que sirven de indicadores relativamente estables de los roles, comportamientos y métodos preferidos por cada profesor. Así mismo, el estilo de enseñanza resalta la relación dinámica que existe entre las concepciones que tienen los profesores acerca de lo que debe ser la educación (concepción idealista de la práctica educativa) y lo que es (actuaciones educativas concretas).

A este respecto, señalamos la existencia histórica de dos grandes estilos: el estilo de enseñanza académico y el estilo de enseñanza activo que según el momento y el contexto fueron adoptando diferentes denominacio- 
nes. Así podemos señalar las experiencias realizadas por Anderson, 1945 y Flanders, 1984 y más recientemente las llevadas a cabo por Coldren y Hively, 2009; Hood, Poulson, Mason, Walker y Dixon, 2009; Gargallo, 2008; Michel, James y Varela, 2009 y Weltman y Whiteside, 2010.

Siguiendo este enfoque, Gargallo (2010) da cuenta de la existencia de diversas investigaciones que abordan los dos grandes componentes «el modelo de «transmisión del conocimiento» o "centrado en la enseñanza», también denominado «modelo centrado en el profesor», y el modelo de «facilitación del aprendizaje», o «centrado en el aprendizaje», que también llamado «modelo centrado en el alumno».

Como ya sabemos, la concepción académica o tradicional, está fundamentada en el discurso expositivo que transmite hechos y conceptos, vinculada a la capacidad de impartir una disciplina y lograr un clima de máximo rigor, rigidez y control. En este caso la enseñanza gira en torno a la figura del profesor (Delgado, 1991, 1994; Martín-Cuadrado, 2011; González-Peiteado, 2013).

Sin embargo, la concepción activa genera un estilo de enseñanza ligado a planteamientos que consideramos más innovadores y a expectativas de éxito y calidad respetando el ritmo de aprendizaje del alumno. Así mismo, se promueven actitudes de cooperación, tolerancia y se fomenta competencias vinculadas al aprender a aprender, a aprender a ser, aprender a hacer y aprender a convivir (Monereo y Pozo, 2003; Samuelowicz y Bain, 2002). A lo que añadimos la importancia de la «reflexión en la acción» hecho que abarca el «conocimiento en la acción» aquél que se revela en las acciones inteligentes, ya sean observables al exterior o que se den internamente en las personas. En ambos casos el conocimiento está en la acción, se evidencia a través de la ejecución espontánea y hábil» (Tejada Fernández, 2011, p. 23).

Para Ballesta, Izquierdo y Romero (2011, p. 358) «los resultados en la enseñanza son mucho más claros cuando los profesores, además de tener una concepción centrada en el aprendizaje, y de usar metodologías coherentes con ella, disponen de más habilidades docentes».

Evidentemente, en las aulas no se dan estos comportamientos en toda su amplitud, sino que el profesor se mueve dentro de un continuum, combinando rasgos de ambos estilos, aunque uno de ellos sea el predominante (González-Peiteado y Aznar, 2010).

Desde el estudio de las diferencias individuales y partiendo de un marco teórico multidimensional, nuestro objetivo es describir y analizar los estilos de enseñanza preferidos por los futuros maestros de la Comunidad de Galicia, identificando sus potencialidades y deficiencias. 


\section{MÉTODO}

Con la pretensión de conocer e interpretar una realidad y de abrir un debate sobre la optimización de la práctica docente y la mejora de los procesos formativos, hemos optado por un diseño no experimental de carácter transversal. Para tal fin, se emplea una muestra no probabilística de sujetos voluntarios, con garantías de aleatoriedad e independencia de los pares de sujetos. La recogida de información se solicitó a los estudiantes a través de un instrumento que incluye datos de identificación y una escala sobre estilos de enseñanza así como la realización de entrevistas semiestructuradas y grupos de discusión.

En esta investigación hemos rentabilizado los aportes de diferentes paradigmas (cualitativo y cuantitativo) a través de una visión plural que nos permite abordar la triangulación de técnicas y de fuentes de recogida de datos, de acuerdo con estudios similares (Pino, Domínguez y López, 2007), o lo referido por Rodríguez (2005) con la intención de conocer la propensión que manifiestan en relación a los estilos de enseñanza.

Con mucha frecuencia en la medición de una creencia o una actitud se comenten involuntariamente sesgos y errores. En consecuencia, con el propósito de evitar alteraciones en los resultados, hemos optado por la triangulación de datos ya que nos permite establecer comparaciones desde diferentes posiciones, precisar consonancias y disonancias, y determinar elementos que permitan decidir sobre la credibilidad de la información.

En última instancia, nuestro propósito radica en la evaluación de los estilos de enseñanza de los futuros docentes de la forma más objetiva posible. En cuanto al contenido, por razones obvias, en esta muestra evaluamos las intenciones u opiniones de los estudiantes en relación con el quehacer docente en lugar de prácticas concretas. Entendiendo por opinión, la postura más o menos constante en el tiempo y representativa de un posicionamiento coherente y una perspectiva consciente sobre cómo entiende la realidad educativa. Por tanto, somos conocedores de la existencia de una mayor implicación de un componente teórico-idealizador sobre el prácticorealista en la etapa de la formación inicial del profesorado que se ha tenido en cuenta en la construcción de la escala.

\section{Sujetos}

En la presente investigación, la población estudiada está constituida por 1314 estudiantes de tercer curso de Magisterio que realizan sus estudios en las tres universidades gallegas. De ellos, 464 pertenecen a Educación 
Infantil, 449 corresponden a Educación Primaria, 148 a Educación Física, 121 están en Educación Musical y 132 son alumnos de Lengua Extranjera.

Teniendo en cuenta el tamaño de la población, hemos obtenido un tamaño muestral aconsejable de $\mathrm{N}=492$ con la ayuda del programa Raossoft (Sample Size Calculator). Dado la cifra señalada, se confirma que la muestra del estudio $(\mathrm{N}=555)$ es suficientemente amplia.

Por tanto, la muestra queda compuesta por 555 estudiantes $(\mathrm{N}=555)$ de formación inicial del profesorado que cursan sus estudios en las tres universidades gallegas: Universidad de Vigo, Universidad de Santiago de Compostela y Universidad de A Coruña.

En función de la variable especialidad, el grupo investigado está compuesto en un $41,3 \%$ por estudiantes de Educación Infantil, mientras que el 29,5\% pertenece a Educación Primaria. Con un porcentaje menor de estudiantes se encuentran las especialidades de Educación Física, 10,8\%; Educación Musical, 7,4\% y, finalmente, Lengua Extranjera con un 11,0\% del total de la muestra. En todo caso, se ha conseguido la existencia de representatividad en las tres Universidades gallegas.

En lo referente al sexo, existe una mayoría de mujeres $(81,4 \%)$ respecto a los hombres $(18,6 \%)$, que lejos de ser un sesgo en esta muestra, es coherente con los patrones ordinarios de presencia de hombres y mujeres en las aulas de Magisterio.

La distribución por edad es la siguiente: el 35,9\% de la muestra tiene menos de 20 años, el 45,9\% integra la franja de 21 a 23 años, mientras que el $18,2 \%$ posee más de 24 años. Este dato también coincide con los patrones tipo del estudiante de Magisterio en nuestro país.

\begin{tabular}{|l|l|l|l|l|}
\hline \multicolumn{1}{|c|}{ Datos } & Categorías & Dimensiones & Frecuencias & Porcentajes \\
\hline \multirow{4}{*}{$\begin{array}{l}\text { Datos } \\
\text { académicos }\end{array}$} & UNIVERSIDAD & U.Vigo & 332 & $59,8 \%$ \\
\cline { 3 - 5 } & & USC & 159 & $28,6 \%$ \\
\cline { 3 - 5 } & U.Coruña & 64 & $11,5 \%$ \\
\cline { 3 - 5 } & Total & 555 & $100 \%$ \\
\cline { 3 - 5 } & ESPECIALIDAD & Ed. Infantil & 229 & $41,3 \%$ \\
\cline { 3 - 5 } & Ed. Primaria & 164 & $29,5 \%$ \\
\cline { 3 - 5 } & Ed. Física & 60 & $10,8 \%$ \\
\hline & Ed. Musical & 41 & $7,4 \%$ \\
\hline & L. Extranjera & 61 & $11,0 \%$ \\
\hline
\end{tabular}




\begin{tabular}{|c|l|l|l|l|}
\hline \multicolumn{1}{|c|}{ Datos } & \multicolumn{1}{|c|}{ Categorías } & Dimensiones & Frecuencias & Porcentajes \\
\hline \multirow{3}{*}{ Datos personales } & \multirow{2}{*}{ SEXO } & Hombre & 103 & $18,6 \%$ \\
\cline { 3 - 5 } & & Mujer & 452 & $81,4 \%$ \\
\cline { 2 - 5 } & \multirow{2}{*}{ EDAD } & Menos de 20 & 199 & $35,9 \%$ \\
\cline { 3 - 5 } & & $21-23$ & 255 & $45,9 \%$ \\
\cline { 3 - 5 } & & 24 o más & 101 & $18,2 \%$ \\
\hline
\end{tabular}

Tabla 1. Datos personales y académicos de la muestra

\section{Instrumentos}

Con la intención de contribuir al propósito de la investigación, se ha realizado una primera aproximación a las concepciones que el alumno manifiesta sobre los estilos de enseñanza, en el último año de carrera, a partir de los elementos simbólicos, ideas y representaciones asociadas a él y manifestadas en los grupos de discusión. Todo ello en línea con propuestas como la de Smith (1988) quien sugiere el uso de metodologías cualitativas para la medición de estilos de enseñanza. De esta forma, en primer lugar, hemos llevado a cabo un estudio exploratorio-descriptivo a través de la configuración de dos grupos de discusión integrados por cinco estudiantes cada uno. Situación que añade un elemento de intensidad y profundidad, para poder integrar sus argumentos posteriormente en un constructo global que permita un mejor conocimiento de lo que acontece. En consecuencia, algunos de los razonamientos realizados por los alumnos pasaron a completar la escala.

En efecto, en segundo término y dentro del espectro de instrumentos de signo cuantitativo empleado en la evaluación de preferencias en el estilo docente hemos elaborado una la escala (Likert, 1953) sobre estilos de enseñanza (ESEE). La finalidad es abarcar, con los 31 ítems que recoge la escala, las principales dimensiones del constructo que se quiere medir, recogiendo cuestiones de interés en la actuación educativa: comunicación, trabajo grupal, participación, atención individualizada, innovación y reflexión, entre otros aspectos. Es así como nos aproximamos al conocimiento del estilo de enseñanza preferido de la muestra estudiada. Con tal fin, hemos revisado los principales instrumentos elaborados por diversos autores a lo largo de los años para la medición de estilos de enseñanza (Delgado, 1991; DomenechBetoret, 2012). Cada enunciado ha sido presentado seguido de una escala de estimación que oscila desde el total acuerdo «siempre» al total desacuerdo «nunca» incluyendo estadios intermedios («frecuentemente», «raras veces») en relación con la proposición. 
En tercer lugar y teniendo en cuenta el criterio de saturación y representación de los diversos grupos estudiados, se han realizado cinco entrevistas a los alumnos de cada una de las especialidades de Magisterio de las tres Universidades $(\mathrm{N}=50)$. Ello ha servido para contrastar cuestiones que se trataban en los instrumentos complementarios (grupo de discusión y escala) con el fin de obtener resultados desde diversas perspectivas y de este modo saturar la información sobre los estilos de enseñanza.

En vista de las apreciaciones precedentes, las preguntas elaboradas dieron respuesta a los objetivos y cuestiones planteadas en la investigación, formuladas en un lenguaje claro, con la intención de conseguir información espontánea e interesante para el estudio. En este sentido, Stake (1998, p. 25) afirma que "Quizá la tarea más difícil del investigador es la de diseñar buenas preguntas, las preguntas de la investigación, que dirijan la atención y el pensamiento lo suficiente pero no en exceso".

En todo caso, se ha asegurado el tratamiento de los temas clave.

\section{Análisis realizados}

- En relación a los grupos de discusión.

Se han tenido presentes dos premisas esenciales: a) No sesgar el proceso comunicativo evitando influir en la línea discursiva, b) Crear un espacio de colaboración en el que los asistentes expresen sus opiniones libremente.

A partir de estos criterios, hemos formulado cinco cuestiones abiertas encaminadas a propiciar la interacción dialógica: ¿Qué entienden por estilo de enseñanza? ¿Consideran que el estilo de enseñanza puede incidir en el correcto aprendizaje del alumno? ¿Qué comportamientos del docente creen que son más eficaces para producir mejoras en los aprendizajes de los alumnos? Si un docente enseña bien ¿por qué hay niños que sin evidenciar trastornos de aprendizaje no aprenden? ¿Cómo caracterizaría su estilo de enseñanza?

En cuanto al análisis de contenido, lo llevamos a cabo en términos descriptivos, conduciendo a la elaboración de informes en los que el material recogido se estructura y sistematiza en torno a una serie de temas considerados relevantes para la elaboración de la escala. Hemos considerado todas las expresiones aportadas aprehendiendo las ideas claves del discurso en torno a dos grandes descriptores: a) La realidad de los estudiantes de Magisterio en torno a la construcción de su propio estilo, b) Concepciones en torno a la aplicación de los estilos de enseñanza en las aulas. 
- En relación a la escala.

La formulación de los elementos de la escala se ha llevado a cabo integrando las aportaciones de diversas fuentes literarias y las ideas expresadas en los grupos de discusión. Proceso que va a ser seguido de una selección de ítems y del estudio de las propiedades psicométricas (González-Peiteado, López-Castedo, Pino-Juste, 2013).

Inicialmente se procedió a la validación de la escala mediante un estudio analítico de dicho instrumento, con el fin de depurar su longitud, teniendo en cuenta los siguientes criterios (Cea, D'Ancona, 1999; Mc Millan y Schumacher, 2005): a) Se eliminan ítems redundantes, con doble sentido o compuestos, b) Es valorado por tres expertos universitarios.

En referencia a las características técnicas del cuestionario, y como paso previo a la selección del método de extracción, se han solicitado dos indicadores de adecuación muestral.

a) El test de Esfericidad de Barlett (TEB) con el fin de determinar si existen correlaciones significativas. El valor obtenido es de 2264,064 con un nivel de significación (p10,00), por tanto existe un nivel de correlación suficiente entre variables

b) El test Kaiser-Meyer-Olkin (KMO) que revela un valor de 0,862, lo que refleja una notable proporción de varianza común entre las variables.

Seguidamente, se ha llevado a cabo un análisis factorial de Componentes Principales con rotación Varimax, con el propósito de confirmar la existencia de las dimensiones fijadas a priori y así poder afirmar que se está midiendo lo que se ha querido medir. Dicho análisis factorial confirma la existencia de dos factores, que en su conjunto explican el $53,260 \%$ de la varianza total de los resultados. Para facilitar su interpretación se recogen sólo las saturaciones superiores a 0.40 . Ambas dimensiones se corresponden con el estilo de enseñanza activo o centrado en el alumno y el estilo de enseñanza académico o centrado en el profesor. A su vez, el estilo de enseñanza activo está integrado por cinco subdimensiones (reflexivo, cooperador, individualizado, innovador e indagador).

Así mismo, con el objetivo de valorar el grado de precisión de la medida, se calculó el coeficiente alfa de Cronbach, obteniendo una alta consistencia interna con un coeficiente de 0,886 para el conjunto de la escala. Con relación a cada uno de los factores, los resultados fueron los siguientes: estilo activo $(\alpha=0,894)$, estilo académico $(\alpha=0,791)$. 
- En relación a las entrevistas.

Con el fin de asegurar el tratamiento de los temas claves y saturar la información recibida, se ha elaborado un protocolo estructurado con preguntas amplias que facilitan que los estudiantes produzcan abundantes valoraciones sobre cada uno de los cuatro aspectos a tratar: ¿Cómo describiría su estilo de enseñanza? ¿Si pudiera diseñar un profesor ideal ¿Cómo sería? ¿Por qué? ¿Qué comportamientos docentes pueden derivar en fracaso escolar? ¿Qué comportamientos docentes estimulan el aprendizaje?

La fase de análisis de contenido consistió en la codificación a mano de palabras clave extraídas de las respuestas de cada sujeto. Hemos definido las actitudes que son relevantes para el estudio y que consideramos que deben ser categorizadas. En una siguiente fase se clasificó a los contenidos por asociación temática. Esto permitió agrupar en categorías las palabras con el mismo significado semántico, lo que llevó a la obtención de 14 indicadores para posteriormente establecer sus frecuencias (ver anexo 2).

\section{Procedimiento}

Como ya hemos mencionado, para alcanzar los objetivos del estudio y con el deseo de evitar sesgos y errores hemos optado por una triangulación de instrumentos. Con la intención básica de contribuir a este propósito, se ha realizado una primera aproximación a las representaciones y percepciones de los estudiantes de Magisterio sobre los estilos de enseñanza a través de la organización de dos grupos de discusión compuesto por cinco estudiantes.

A partir del análisis del contenido emergente en estas reuniones y del estudio intensivo de la bibliografía existente, hemos recogido contribuciones importantes para la elaboración de la escala ESEE, sometida al estudio de los datos obtenidos mediante el paquete estadístico SPSS 19.0. Así mismo, hemos querido ampliar el carácter informativo-descriptivo de esta parte de la investigación con el uso ulterior de la entrevista a alumnos de las distintas especialidades.

Todos los sujetos participaron de forma voluntaria y anónima en el estudio y se respetaron todos los procedimientos éticos para la recogida de datos.

\section{RESULTADOS}

Se han organizado los resultados obtenidos describiéndolos de forma sistemática en función de los dos factores (estilo de enseñanza académico 
y estilo de enseñanza activo) que han emergido de la estructura factorial de la escala en el estudio realizado previamente sobre sus propiedades psicométricas.

El análisis de los datos se ha efectuado en dos niveles. En primera instancia, se lleva a cabo un estudio descriptivo de las dimensiones objeto de estudio en función de tres variables: especialidad, sexo y edad. A continuación, se realiza un análisis inferencial con la intención de determinar si existen diferencias significativas entre las distintas categorías. Con tal propósito, hemos integrado todo el trabajo de campo en un todo coherente con el objetivo de obtener resultados veraces y contrastados a partir de la integración de instrumentos de corte cuantitativo (escala de estilos de enseñanza) y de signo cualitativo (entrevista y grupo de discusión).

\section{Análisis descriptivo}

En una primera aproximación a los resultados observamos que el estilo activo es el preferido por los estudiantes siendo la especialidad de Lengua Extranjera (85.02\%) y Educación Infantil (83.20\%) las que puntúan más alto en este estilo, mientras que la de Educación Física (74.62\%) es la que genera menos evaluaciones positivas en relación al estilo activo.

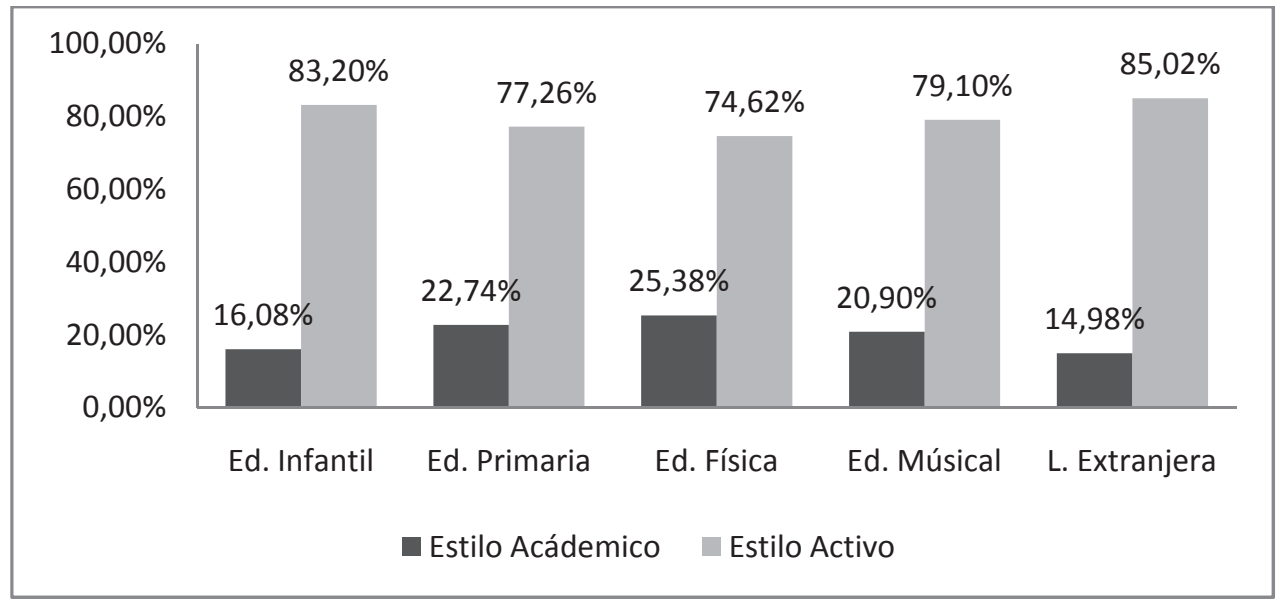

Gráfico 1. Estilos de enseñanza en función de la especialidad de la muestra

Del análisis efectuado a partir de los argumentos aportados en las entrevistas y grupos de discusión, hemos constatado claramente como los alumnos de Educación Física denotaban una mayor inclinación hacia el estilo académico en comparación con las creencias apuntadas por los in- 
tegrantes de las otras especialidades. Sin embargo, este apunte hacia un estilo académico, concretamente «mando directo», se combina con el uso de estilos activos como veremos a continuación en dos comentarios recogidos por ser especialmente ilustrativos:

«Creo que el estilo de enseñanza ideal es el que fusiona mando directo, autoaprendizaje y trabajo cooperativo» (Ed.Física1).

«Combinaría la asignación de tareas más mando directo, pero en general una mezcla de estilos para favorecer una mayor participación» (Ed.Física3).

Además hemos de señalar que las dos dimensiones descritas (enseñanza académica versus enseñanza activa) arrojaron diferencias con respecto al sexo. Así, los hombres en comparación con las mujeres, manifiestan una mayor tendencia hacia estilos tradicionales. Mientras que solamente el $18.20 \%$ de las mujeres presentan una tendencia hacia el estilo tradicional y un $81.80 \%$ hacia el estilo activo; el $26,18 \%$ de hombres se identifica con el estilo tradicional y el $73.82 \%$ lo hace con el estilo activo. Es decir, las futuras docentes que participaron en este estudio rechazan más que los hombres el estilo tradicional.

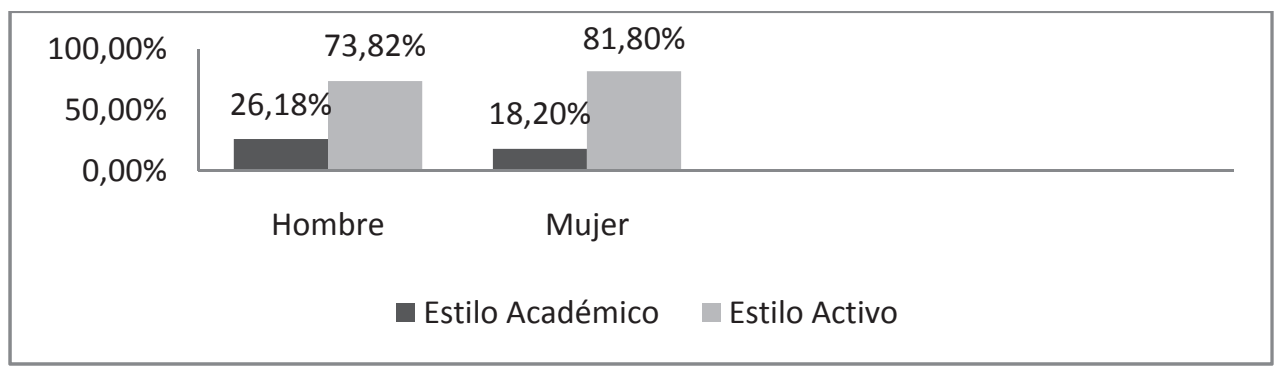

Gráfico 2. Estilos de enseñanza en función del sexo de la muestra

En relación a esta dimensión, en las entrevistas y grupos de discusión se pone de manifiesto una tendencia concordante con los datos aportados por la escala. Destacamos a modo de ejemplo algunos argumentos manifestados en los grupos de discusión:

"Es importante que sepa transmitir conocimientos y que sea disciplinado (...) Personalmente combinaría la asignación de tareas con el mando directo» (G2. Hombre, Ed Física). 
"Me identifico con un profesor que se interesa por su asignatura y en como transmitirla a los alumnos para que ellos adquieran conocimientos.»(G2. Hombre, L.Extranjera).

Las razones esgrimidas por estos estudiantes nos hacen retrotraernos hacia períodos históricos donde lo fundamental eran los «conocimientos» y su «correcta transmisión». Sin embargo, se hace preciso destacar que los comentarios relacionados con un estilo tradicional-academicista figuran con escasa frecuencia en los discursos, por lo menos en su vertiente más radical.

A continuación vemos como, en el transcurso de los argumentos aportados, se articulan ejes conceptuales y actitudes favorables a la incorporación de estilos de enseñanza activos que impliquen al alumno:

"Es importante favorecer el aprendizaje por descubrimiento por parte del alumno, creo que es necesario que descubran poco a poco los fines que buscamos para que aprendan a trabajar y buscar la información, pero también a trabajar en grupo y a valorar el esfuerzo personal. Aunque finalmente sea yo quien les dé la solución para que comparen lo real con lo que han hecho y corrijan sus errores (...) muchas veces el profesor desanima al alumno. Si el alumno no alcanza los conocimientos hay que ayudarle a conseguir los objetivos a través de distintos métodos que le ayuden» (G1.Mujer Ed.Infantil).

"A mí me gustaría que los alumnos comprendieran la importancia de descubrir cosas, mediante la motivación y las ganas de aprender. Para ello sería positivo que pudieran ayudarse unos a otros, participando en trabajos grupales que le sirvan para interiorizar mejor los conceptos (...) creo que eso se consigue mirando con esperanza a los alumnos, tratando de dar explicaciones claras y preocupándonos por poner en práctica la teoría todos los días y en la medida de lo posible.» (G2. Mujer. Ed.Primaria.).

En efecto, todas las argumentaciones expresadas han consolidado un mayor conocimiento acerca de las percepciones de los estudiantes sobre el hecho educativo. Sin embargo, en todo caso, hemos tenido en cuenta que el factor de deseabilidad social podría ocultar en ciertos momentos la expresión sincera de cuál sería su comportamiento real en el aula.

En relación con la edad, los resultados obtenidos en la escala confirman un patrón ascendente hacia los estilos de enseñanza activos. Es decir, cuanto mayor es la edad del alumnado mayor es la inclinación hacia estilos más activos. 


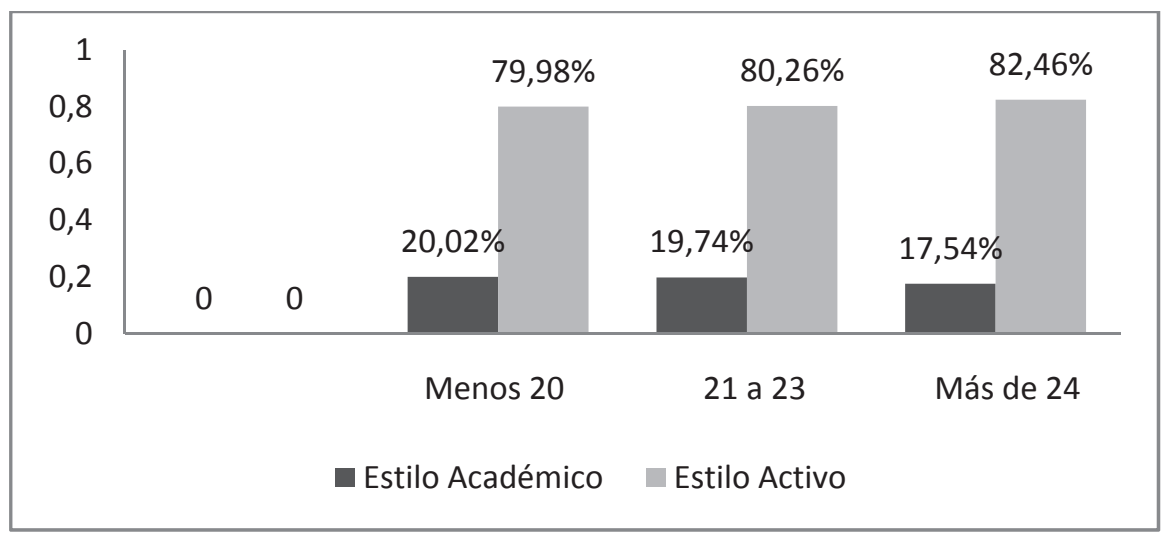

Gráfico 3. Estilos de enseñanza en función de la edad de la muestra

Esta tendencia no se ha podido constatar en el análisis de contenido de los textos fruto de los grupos de discusión o las entrevistas.

\section{Análisis inferencial}

El análisis inferencial se llevó a cabo bajo tres situaciones. Para las variables especialidad y edad se ha aplicado un diseño multigrupo con el propósito de verificar la relación funcional entre estas categorías y los estilos de enseñanza utilizando el análisis de varianza (ANOVA) de un factor. En la tabla 2 y 3 se recoge el análisis de varianza en función de la especialidad y la edad. Los resultados muestran que ambas dimensiones, son fuentes significativas de variación, por lo que se deriva la existencia de un estilo preferente en función de la especialidad que se curse.

Los resultados confirman que los integrantes de la muestra que cursan Educación Infantil poseen en mayor medida creencias coherentes con estilos de enseñanza activos, estableciendo diferencias significativas con los estudiantes de Educación Física y Educación Primaria. Le suceden los estudiantes de Lengua Extranjera, existiendo diferencias significativas con los alumnos de Educación Física, quienes en la muestra recogida son los que manifiestan una percepción menos proclive a estilos activos.

Atendiendo al estilo académico, observamos que los integrantes de la muestra de Educación Infantil se revelan como significativamente menos tradicionales en relación con los estudiantes de Educación Primaria y Educación Física. Seguido de los alumnos de Educación Musical y Lengua Extranjera. Siendo el alumnado de Educación Física el que presenta una mayor propensión hacia el estilo académico. 


\begin{tabular}{|c|c|c|c|c|}
\hline FACTORES & Media & $\mathrm{F}$ & Sig. & HSD de TUKEY Sig.* \\
\hline \multirow[t]{5}{*}{ Estilo Activo } & Ed. Infantil: 85,80 & \multirow[t]{5}{*}{12,00} & \multirow[t]{5}{*}{0,000} & \multirow{5}{*}{$\begin{array}{l}\text { Ed.Infantil-Ed.Primaria: } \\
\text { 0,000 } \\
\text { Ed.Infantil-Ed.Física:0,000 } \\
\text { Ed Física-L.Extranjera: } \\
\text { 0,006 }\end{array}$} \\
\hline & Ed. Primaria: 82,06 & & & \\
\hline & Ed. Física: 79,87 & & & \\
\hline & Ed. Musical: 83,35 & & & \\
\hline & Ed.Extranjera: 84,24 & & & \\
\hline \multirow{5}{*}{$\begin{array}{l}\text { Estilo Aca- } \\
\text { démico }\end{array}$} & Ed. Infantil:11,93 & \multirow[t]{5}{*}{10,30} & \multirow[t]{5}{*}{0,000} & \multirow{5}{*}{$\begin{array}{l}\text { Ed.Infantil-Ed.Primaria: } \\
\text { 0,000 } \\
\text { Ed.Infantil-Ed.Física: 0,000 } \\
\text { Ed.Física-Ed.musical: 0,014 } \\
\text { Ed.Física-L.Extranjera: } \\
\text { 0,026 }\end{array}$} \\
\hline & Ed. Primaria:13,58 & & & \\
\hline & Ed. Física:14,38 & & & \\
\hline & Ed. Musical:12,30 & & & \\
\hline & Ed.Extranjera:12,62 & & & \\
\hline
\end{tabular}

* La diferencia de medias es significativa al nivel .005

Tabla 2. Análisis de varianza y HSD de Tukey por especialidad

$\mathrm{Al}$ analizar si la edad influye en la configuración de los estilos de enseñanza en la muestra estudiada, hemos concluido que efectivamente existen diferencias en torno al estilo activo entre los rangos comprendidos entre 21-23 años y más de 24 años. Siendo estos últimos los que evidencian una mayor inclinación hacia estilos activos.

\begin{tabular}{|c|c|c|c|c|}
\hline FACTORES & Media & $\mathrm{F}$ & Sig. & HSD de TUKEY Sig. \\
\hline \multirow[t]{3}{*}{ Estilo Activo } & 18-20 años: 83,73 & \multirow[t]{3}{*}{3,324} & \multirow[t]{3}{*}{0,037} & \multirow{3}{*}{$\begin{array}{l}\text { *Diferencias entre } 21-23 \text { y } \\
24 \text { o más = sig } 0,029\end{array}$} \\
\hline & 21-23 años: 83,06 & & & \\
\hline & 24 o más: 85,24 & & & \\
\hline \multirow[t]{3}{*}{ Estilo académico } & 18-20 años: 12,88 & \multirow[t]{3}{*}{1,145} & \multirow[t]{3}{*}{0,319} & \\
\hline & 21-23 años: 12,89 & & & \\
\hline & 24 o más: 12,33 & & & \\
\hline
\end{tabular}

* La diferencia de medias es significativa al nivel .05

Tabla 3. Análisis de varianza y HSD de Tukey por edad

Para la variable sexo, hemos utilizado un diseño unifactorial intersujetos. En consecuencia, mediante la diferencia de medias (prueba t) interpretamos si las medias correspondientes a dos grupos difieren de manera significativa. De esta forma, hemos corroborado la existencia de diferencias significativas entre los sexos en las dos dimensiones estudiadas. En la Tabla 4 se muestran las medias, la prueba t y el nivel de significación, sobre cómo 
se comportan los estilos en función del sexo. En vista de los resultados se concluye que el sexo influye en la conformación de un estilo de enseñanza preferente.

\begin{tabular}{|l|l|c|c|}
\hline \multicolumn{1}{|c|}{ FACTORES } & \multicolumn{1}{|c|}{ Media } & T & Sig. \\
\hline \multirow{2}{*}{ Estilo Activo } & Hombre: 79,91 & $-6,028$ & 0,000 \\
\cline { 2 - 2 } & Mujer : 84,55 & & \\
\hline Estilo Académico & Hombre: 14,09 & 4,414 & 0,000 \\
\cline { 2 - 2 } & Mujer: 12,49 & & \\
\hline
\end{tabular}

Tabla 4. Prueba t de Student para el contraste de medias de muestras independientes

\section{CONCLUSIONES Y DISCUSIÓN}

Dada la escasez de investigaciones sobre las creencias y opiniones de los futuros maestros en torno al estilo de enseñanza a adoptar en su función docente, este estudio ha buscado la complementariedad entre los paradigmas cualitativo y cuantitativo, con el propósito de precisar, describir y comprender una realidad. Todo ello, a través de un marco metodológico que enriquece el estudio, capitalizando las contribuciones de diferentes técnicas y fuentes de recogida de datos, para así poder comparar y comprobar la exactitud de los aportes conseguidos.

La credibilidad de esta investigación se sustenta en el hecho de que el objeto de estudio, "conocer las representaciones y creencias que sobre los estilos de enseñanza tienen los alumnos del último curso de Magisterio en la Comunidad de Galicia», fue identificado y descrito a través de las propias manifestaciones y juicios críticos de los protagonistas de las tres universidades, y por tanto, los comportamientos descritos y analizados son los que realmente existen en los contextos señalados. Esta credibilidad también está reflejada en la triangulación de datos, de perspectivas, e instrumentos de recogida de información con la intención de conseguir la corroboración de testimonios y la coherencia estructural con respecto a las creencias del alumnado y realizando diferentes comprobaciones a lo largo del estudio.

Como principal conclusión podemos afirmar que existe un predominio, en el discurso de los futuros docentes, de un vocabulario ligado a concepciones activas y humanistas unidas al corpus defendido por los teóricos de la educación. A propósito de lo referido anteriormente, Delgado, Medina y Viciana (1996) han investigado sobre la preferencia de los estilos de enseñanza de los estudiantes de formación inicial del profesorado de la especia- 
lidad de Educación Física. Los resultados indican una clara tendencia hacia estilos activos y una valoración negativa hacia estilos tradicionales. En un estudio posterior, Delgado (1998) analiza los estilos de enseñanza en estudiantes en formación inicial de Educación Física y profesores en ejercicio de su profesión. En ambos casos hay un manifiesto predominio de los estilos activos frente a los estilos tradicionales.

Esta tendencia hacia estilos centrados en el alumno es más acusada entre los futuros profesores de Educación Infantil. Evidentemente, no podemos afirmar, a partir de los datos recogidos, que las creencias positivas hacia estilos activos signifique una apropiación sustantiva de este comportamiento en sus futuras prácticas educativas. Sin embargo, al no manifestarse contradicciones en el discurso de los estudiantes, podemos asegurar que, en su mayoría, han interiorizado la importancia de adoptar estilos de enseñanza activos, dinamizadores y coherentes con los principios de individualización, cooperación y enseñanza reflexiva, entre otros.

Por otro lado, la realidad nos muestra que los profesores de Educación Infantil han sido pioneros en el diseño de proyectos de innovación educativa y en la intervención educativa que realizan suelen utilizar técnicas y métodos activos de aprendizaje.

Convenimos en que en las exposiciones, los estudiantes no evidencian el agotamiento de un estilo de enseñanza academicista sustentado en la transmisión de conocimientos. Sin embargo, las características que configuran principalmente su representación de la práctica educativa se basa fundamentalmente en un estilo activo de la enseñanza. Podemos observar que, en modo alguno, el marco pedagógico de la formación docente mantiene configuraciones didácticas que repiten aspectos del modelo tradicional, que justamente se intentan transformar. Más bien descubrimos la existencia de una asunción de un estilo mixto de enseñanza, el cual consideran conveniente para atender las necesidades educativas del alumnado y los distintos ritmos y estilos de aprendizaje.

En consecuencia, el presente estudio nos ha brindado los soportes sustanciales para identificar las creencias y los núcleos teóricos en los que se apoyan las imágenes que poseen los estudiantes en formación acerca del acto educativo y sus vicisitudes, lo que tiene múltiples implicaciones teóricas, metodológicas y prácticas. Nuestro propósito es, a partir del análisis de la situación real en torno a la percepción de los propios estilos educativos, ayudar a los futuros docentes a realizar un análisis reflexivo y crítico sobre su forma de actuar y pensar en relación con el proceso educativo. Circunstancia que aporta información con alcance para la investigación, así como para los alumnos de Magisterio y profesionales de la educación. 
Dado que parece que los resultados muestran que los futuros profesores optan por estilos activos de enseñanza, futuras investigaciones deberían evaluar otras variables como la influencia de la organización del centro en la configuración de los estilos, los factores institucionales que inciden en la adquisición de una tendencia determinada: tamaño grupo-clase, recursos, legislación educativa, entre otros. En todo caso, confiamos en que los resultados encontrados hasta ahora y los futuros ayudarán a diseñar programas más eficaces de formación inicial del profesorado y una intervención educativa más precisa. Como apunta Martín-Cuadrado y Sánchez-Elvira (2010) las características individuales y el comportamiento humano no son rasgos inamovibles, por lo que se pueden modificar las actuaciones que resultan ineficientes y entrenar aquellas que son convenientes para el buen desempeño de la tarea educativa.

En última instancia y como síntesis de las ideas expuestas destacamos el pensamiento de Román (2008, p. 32): «si enseñar es ayudar a aprender, y para aprender hay que estar mentalmente activos, el maestro debe ayudar a aprender utilizando una serie de habilidades docentes básicas y motivadoras. Habilidades que concebimos como, todas aquellas acciones, conductas, actitudes y patrones de comportamiento implicados habitualmente en el aula, que se presentan íntimamente ligadas a las diferentes competencias, estilos de enseñanza-aprendizaje, y a las propias funciones y finalidades de la enseñanza».

De ahí la importancia de precisar cuáles son las razones por las cuáles los profesores manifiestan reiteradamente modelos tradicionales en el aula, si su pensamiento y actitudes están más dirigidas a modelos activos. 


\section{REFERENCIAS BIBLIOGRÁFICAS}

Anderson, H.H. (1945). Domination and social integration in the behavior of kindergarten children and teachers. Genetic psychological monographs, 21, 287-385.

Ballesta, F; Izquierdo, T y Romero, B. (2011). Percepción del alumnado de Pedagogía ante el uso de metodologías activas. Educatio Siglo XXI, 29 (2), 353-368.

Blanco Felip, F.; Jové Deltell, M.C. y Reverter Masià, J. (2012). Paradigma estratégico para el desarrollo de habilidades competenciales. Estudio descriptivo sobre la variabilidad en la percepción de habilidades competenciales de 40 alumnos de educación física en fase de formación inicial. Educación XX1, 15 (2), 231-248.

Coldren, J. y Hively, J. (2009). Interpersonal teaching style and student impression formation. College Teaching, 57 (2), 93-98.

Delgado, M.A. (1991). Los Estilos de Enseñanza en la Educación Física. Propuesta para una reforma de la enseñanza. Granada: Universidad de Granada.

Delgado, M.A. (1994). Intervención didáctica en Primaria. Implicaciones en la Formación del Maestro especialista. En S. Romero (Comps.). Didáctica de la E.F.: Diseños Curriculares en Primaria (pp. 111-117). Sevilla: Wanceulen.

Delgado, M.A. (1998). Comparación de la valoración de los Estilos de Enseñanza por los futuros profesores de Educación Física durante la Formación Inicial y Profesores de Educación Física en Formación. Lecturas de Educación Física. Recuperado de: http:// w.w.w.sir.ca/revista/
Delgado, M.A.; Medina, J. y Viciana, J. (1996). The Teaching Styles in the Preservice of Physical Education Teachers. International Seminar AIESEP. Lisbon.

Doménech Betoret, F. (2012). Análisis de los estilos de pensamiento que utilizan los profesores españoles en el aula. Revista de Educación. 358, 497-522.

Cea D'ancona, M. A. (1999). Métodos y Técnicas de Investigación Cuantitativa. Madrid: Editorial Síntesis.

Feiman-Nemser, S. (2001). From Preparation to Practice: Designing a Continuum to Strengthen and Sustain Teaching. Teachers College Record, 103(6), 1.013-1.055.

Flanders, N.A. (1984). Análisis de la interacción didáctica. Madrid: Anaya.

Gargallo, B. (2008). Estilos de docencia y evaluación de los profesores universitarios y su influencia sobre los modos de aprender de sus estudiantes. Revista Española de Pedagogía, 241, 425-446.

Gargallo, B.; Sánchez, F.; Ros, C. y Ferreras, A. (2010). Estilos docentes de los profesores universitarios. La percepción de los alumnos de los buenos profesores. Revista Iberoamericana de Educación, 51 (4), 1-16.

González Peiteado, M. (2010). Estilos de enseñanza: Un constructo nuclear de gran impacto en la praxis docente. Revista Educación y Futuro Digital. Recuperado de www.cesdonbosco.com/ revista/articulos2010/

González-Peiteado, M. (2013). Los estilos de enseñanza y aprendizaje como soporte de la actividad docente. Revista de estilos de aprendizaje 11, 51-70. Recuperado de http://www.uned.es/re- 
vistaestilosdeaprendizaje/numero_11/ lsr_11_abril_2013.pdf

González-Peiteado, M. y Aznar-Cuadrado, V. (2010) Aproximación al perfil de estilos de enseñanza en la formación inicial del profesorado de Educación Secundaria: nuevos canales, nuevos retos. Actas del I Congreso Internacional Reinventar la profesión docente. (pp. 348-356). Málaga.

González-Peiteado, M. (2012). Las representaciones sobre los estilos de enseñanza en la construcción del rol docente. Tesis doctoral inédita. Universidad de Vigo.

González-Peiteado, M.; Lopez-Castedo, A. y Pino-Juste, M. (2013). Análisis psicométrico de una Escala sobre Estilos de Enseñanza (ESEE). Revista Enseñanza \& Teaching, 31 (1) 181-198.

Hood, J., Poulson, R., Mason, S., Walker, T. y Dixon, J. (2009). An examination of traditional and nontraditional students» evaluations of professorial leardership styles: transformational versus transactional approach. Journal of the Scholarship of Teaching and Learning, 9 (1), 1-12.

Lickert, R. (1953). A technique for the measurement of attitudes. Archives of Psychology, 22 (140), 1-55.

Lozano Rodríguez, A (2004). Estilos de aprendizaje y enseñanza. Sevilla: MAD.

Marcelo García, C. (2009). Formalidad e Informalidad en el Proceso de Aprender a Enseñar. Revista de Educación, 350, 31-35.

Martín-Cuadrado, A.M. (2011). Competencias del estudiante autorregulado y los estilos de aprendizaje. Revista Estilos de Aprendizaje, 8 (8). Recuperado de www.uned.es/revistaestilosdeaprendizaje/...8/.../lsr_8_articulo_8.pdf
Martín-Cuadrado, A. y Sánchez-Elvira, A. (2010). Ser estudiante a distancia, diferencias individuales y aprendizaje autónomo. En Sánchez García, M.F y y otras (Coords) Entrenamiento en competencias para el estudio autorregulado. Madrid: UNED.

McMillan, J. y Schumacher, S. (2005). Investigación Educativa. Madrid: Pearson Educación.

Michel, N., James, J. y Varela, O. (2009). Active versus passive teaching styles: an empirical study of student learning outcomes. Human Resource Development Quarterly, vol. 20 (4), 397-418.

Monereo, C. y Pozo, J.I. (2003). La universidad ante la nueva cultura educativa. Madrid: Síntesis.

Nevot Luna, A y Cuevas Cava, V. (2009). Los estilos de aprendizaje y el Espacio Europeo de Educación Superior. Un paseo por el aula de matemáticas. Revista de Estilos de Aprendizaje 3 (3) 38-56.

Pino, M.; Domínguez, J. y López, A. (2007). Evaluating appreciation of measures attending to pupil diversity (EMAD). Psychological Reports, 100, 783-786.

Rodríguez, O. (2005). La Triangulación como Estrategia de Investigación en Ciencias Sociales. Revista de Investigación en Gestión de la Innovación y Tecnología. 31. Recuperado de http:// www.madrimasd.org/revista/revista31/ tribuna/tribuna2.asp

Román, J. M. (2008). Psicología de la instrucción. Universidad de Valladolid: Departamento de Psicología. Documento mimeografiado.

Samuelowicz, K. y Bain, J.D (2002). Identifying academics» orientatios to assessment practice. Higher Education, 43, 173-201. 
Smith, R. M. (1988). Learning how to learn. Milton Keynes, UK: Open University Press.

Stake, R. E. (1998). Investigación con estudio de casos. Madrid: Ediciones Morata.

Tejada Fernández, J. (2011). La alternancia de contextos para la adquisición de competencias profesionales en escenarios complementarios de Educación
Superior: Marco y estrategia. Educación XX1. 15 (2), 17-40

Weltman, D. y Whiteside, M. (2010). Comparing the Effectiveness of Traditional and Active Learning Methods in Business Statistics: Convergence to the Mean. Journal of Statistics Education 18, 1. Recuperado de http://www. amstat.org/publications/jse/v18n1/weltman.pdf. 


\section{ANEXO 1}

Escala sobre Estilos de Enseñanza (ESEE)

\section{DATOS DE IDENTIFICACIÓN}

1. Universidad de

\begin{tabular}{|l|l|l|l|}
\hline 2. Curso en el que esta matriculado & $1^{\circ}$ & $2^{\circ}$ & $3^{\circ}$ \\
\hline
\end{tabular}

3. Especialidad

\begin{tabular}{|c|c|c|}
\hline 4. Sexo & Hombre & Mujer \\
\hline
\end{tabular}

\begin{tabular}{|l|l|l|l|l|}
\hline \multicolumn{1}{|c|}{ Grado de pertinencia } & $\begin{array}{c}1 \\
\text { Nunca }\end{array}$ & $\begin{array}{c}2 \\
\text { Raras } \\
\text { veces }\end{array}$ & $\begin{array}{c}3 \\
\text { Frecuente }\end{array}$ & $\begin{array}{c}4 \\
\text { Siempre }\end{array}$ \\
\hline $\begin{array}{l}\text { 1. El verdadero aprendizaje es aquel } \\
\text { que se produce a través de un proce- } \\
\text { so de descubrimiento del alumno. }\end{array}$ & & & & \\
\hline $\begin{array}{l}\text { 2. Favorecer la adquisición de hábitos } \\
\text { de convivencia en grupo y de respeto } \\
\text { a los otros. }\end{array}$ & & & & \\
\hline $\begin{array}{l}\text { 3. Valorar positivamente las aportacio- } \\
\text { nes del alumnado. }\end{array}$ & & & & \\
\hline $\begin{array}{l}\text { 4. Los niños llegarán a los conocimien- } \\
\text { tos a través de la experiencia, de la } \\
\text { búsqueda y el tanteo. }\end{array}$ & & & & \\
\hline $\begin{array}{l}\text { 5. El principal fin de la evaluación es } \\
\text { calificar a los alumnos. }\end{array}$ & & & \\
\hline $\begin{array}{l}\text { 6. Hay que fomentar actividades que re- } \\
\text { quieran cooperación y colaboración. }\end{array}$ & & & & \\
\hline $\begin{array}{l}\text { 7. El docente debe ser original e imagi- } \\
\text { nativo. }\end{array}$ & & & & \\
\hline $\begin{array}{l}\text { 8. Ofrecer a los estudiantes la oportuni- } \\
\text { dad de trabajar con diferentes mate- } \\
\text { riales y bajo diferentes condiciones. }\end{array}$ & & & & \\
\hline $\begin{array}{l}\text { 9. La enseñanza debe girar en torno al } \\
\text { profesor. }\end{array}$ & & & \\
\hline $\begin{array}{l}\text { 10. Importante potenciar la autode- } \\
\text { terminación y al autocontrol del } \\
\text { alumno. }\end{array}$ & & & & \\
\hline
\end{tabular}




\begin{tabular}{|l|l|l|l|l|}
\hline \multicolumn{1}{|c|}{ Grado de pertinencia } & $\begin{array}{c}1 \\
\text { Nunca }\end{array}$ & $\begin{array}{c}2 \\
\text { Raras } \\
\text { veces }\end{array}$ & $\begin{array}{c}3 \\
\text { Frecuente }\end{array}$ & $\begin{array}{c}4 \\
\text { Siempre }\end{array}$ \\
\hline $\begin{array}{l}\text { 11. La evaluación es el único indicador } \\
\text { fiable de la calidad del aprendizaje. }\end{array}$ & & & & \\
\hline $\begin{array}{l}\text { 12. La relación alumnos-padres-profe- } \\
\text { sores deben regular la vida en las } \\
\text { aulas. }\end{array}$ & & & & \\
\hline $\begin{array}{l}\text { 13. Es importante que el educando } \\
\text { sienta como propias las metas pro- } \\
\text { puestas por el grupo. }\end{array}$ & & & & \\
\hline $\begin{array}{l}\text { 14. Hay que favorecer las relaciones in- } \\
\text { terpersonales de los alumnos }\end{array}$ & & & \\
\hline $\begin{array}{l}\text { 15. Los padres deben participar en la } \\
\text { elaboración de los fines educativos } \\
\text { de la formación del alumno. }\end{array}$ & & & \\
\hline $\begin{array}{l}\text { 16. Fomentar la aportación de ideas } \\
\text { nuevas }\end{array}$ & & & \\
\hline $\begin{array}{l}\text { 17. Los objetivos deben tener en cuen- } \\
\text { ta las necesidades e intereses del } \\
\text { alumnado. }\end{array}$ & & & \\
\hline $\begin{array}{l}\text { 18. La enseñanza debe ajustarse al rit- } \\
\text { mo que marque cada niño. }\end{array}$ & & & \\
\hline $\begin{array}{l}\text { 19. La participación del alumno en el } \\
\text { proceso de enseñanza-aprendizaje } \\
\text { motiva al alumno a perseguir los } \\
\text { objetivos fijados. }\end{array}$ & & & \\
\hline $\begin{array}{l}\text { 20. El profesor debe poner más énfasis } \\
\text { en el dominio de conocimientos que } \\
\text { en la adquisición de actitudes. }\end{array}$ & & & \\
\hline $\begin{array}{l}\text { 21. Es mejor que los niños se agrupen } \\
\text { en sectores por su mayor o menor } \\
\text { aptitud. }\end{array}$ & & & \\
\hline $\begin{array}{l}\text { 22. El trabajo en grupo provoca la dis- } \\
\text { persión en el alumno y por tanto el } \\
\text { aprendizaje superficial. }\end{array}$ & & & \\
\hline $\begin{array}{l}\text { 23. Es fundamental estimular a los } \\
\text { alumnos para que aprendan por si } \\
\text { mismos. } \\
\text { menondiande con aquesperados que rinden }\end{array}$ & & & \\
\hline $\begin{array}{l}\text { 24. } \\
\text { may que mantener niveles elevados }\end{array}$ & & & \\
\hline
\end{tabular}




\begin{tabular}{|c|c|c|c|c|}
\hline Grado de pertinencia & $\begin{array}{c}1 \\
\text { Nunca }\end{array}$ & $\begin{array}{c}2 \\
\text { Raras } \\
\text { veces }\end{array}$ & $\begin{array}{c}3 \\
\text { Frecuente }\end{array}$ & $\begin{array}{c}4 \\
\text { Siempre }\end{array}$ \\
\hline $\begin{array}{l}\text { 25. Se deben relacionar los conoci- } \\
\text { mientos de unas materias con otras } \\
\text { cuantas veces sea posible. }\end{array}$ & & & & \\
\hline $\begin{array}{l}\text { 26. Cuando un discente fracasa en una } \\
\text { tarea es importante estimularle a } \\
\text { repensar la actividad y formular } \\
\text { nuevas alternativas de trabajo. }\end{array}$ & & & & \\
\hline $\begin{array}{l}\text { 27. Lo importante es que cada niño in- } \\
\text { tervenga en la búsqueda, discusión, } \\
\text { consulta, aunque esto conlleve más } \\
\text { tiempo. }\end{array}$ & & & & \\
\hline $\begin{array}{l}\text { 28. Es más importante la enseñanza de } \\
\text { técnicas de estudio y de investiga- } \\
\text { ción que la transmisión de un cuer- } \\
\text { po de conocimiento. }\end{array}$ & & & & \\
\hline $\begin{array}{l}\text { 29. Es importante utilizar los agrupa- } \\
\text { mientos de los alumnos como vía } \\
\text { para favorecer la participación en } \\
\text { procesos de aprendizaje. }\end{array}$ & & & & \\
\hline $\begin{array}{l}\text { 30. El docente debe provocar la re- } \\
\text { flexión. }\end{array}$ & & & & \\
\hline $\begin{array}{l}\text { 31. Es necesario motivar a los alumnos } \\
\text { a aprender de forma autónoma }\end{array}$ & & & & \\
\hline
\end{tabular}




\section{ANEXO 2}

Plantilla de categorización de la información de las entrevistas.

\begin{tabular}{|c|c|c|c|c|c|c|c|c|c|c|c|c|c|}
\hline \multirow[t]{2}{*}{ Dimensiones } & \multicolumn{6}{|c|}{ Especialidad } & \multicolumn{3}{|c|}{ Sexo } & \multicolumn{4}{|c|}{ Edad } \\
\hline & $\mathrm{I}$ & $\mathrm{P}$ & M & $\mathrm{EF}$ & LE & $\mathrm{T}$ & $\mathrm{H}$ & M & $\mathrm{T}$ & -20 & \begin{tabular}{|c|}
$21-$ \\
23 \\
\end{tabular} & +20 & $\mathrm{~T}$ \\
\hline \multicolumn{14}{|l|}{ Estimula la reflexión } \\
\hline \multicolumn{14}{|l|}{ Motiva } \\
\hline \multicolumn{14}{|l|}{\begin{tabular}{|l} 
Promueve la capacidad \\
crítica
\end{tabular}} \\
\hline \multicolumn{14}{|c|}{ Fomenta la participación } \\
\hline \multicolumn{14}{|l|}{ Impulsa la socialización } \\
\hline \multicolumn{14}{|l|}{ Favorece la creatividad } \\
\hline \multicolumn{14}{|c|}{\begin{tabular}{|l|} 
Estimula el pensamiento \\
divergente
\end{tabular}} \\
\hline \multicolumn{14}{|l|}{ Es divertido } \\
\hline \multicolumn{14}{|l|}{$\begin{array}{l}\text { Guía el proceso de } \\
\text { aprendizaje }\end{array}$} \\
\hline \multicolumn{14}{|l|}{ Transmisor } \\
\hline \multicolumn{14}{|l|}{$\begin{array}{l}\text { No favorece la } \\
\text { comunicación }\end{array}$} \\
\hline \multicolumn{14}{|l|}{ Excesiva rigidez } \\
\hline \multicolumn{14}{|l|}{\begin{tabular}{|l}
$\begin{array}{l}\text { No individualiza la } \\
\text { enseñanza }\end{array}$ \\
\end{tabular}} \\
\hline $\begin{array}{l}\text { Centrado en la } \\
\text { competencia acadé- } \\
\text { mica }\end{array}$ & & & & & & & & & & & & & \\
\hline
\end{tabular}




\section{PALABRAS CLAVE}

Estilos de enseñanza, investigación educativa, triangulación, creencias.

\section{KEY WORDS}

Teaching styles, educational research, triangulation, beliefs.

\section{PERFIL ACADÉMICO Y PROFESIONAL DE LAS AUTORAS}

Margarita González-Peiteado, Doctora en Ciencias de la Educación por la Universidad de Vigo. Licenciada en Ciencias de la Educación por la UNED.

Ha impartido docencia en la Universidad de Vigo y en la actualidad es profesora-tutora del Centro Asociado de la UNED en Pontevedra.

Sus líneas de investigación están centradas en la Formación Inicial del Profesorado y los Estilos Docentes. Ha participado en Congresos y ha realizado numerosas investigaciones y publicaciones en revistas del ámbito educativo indizadas en prestigiosas bases de datos y con elevado índice de impacto. Igualmente ha participado en varios proyectos sobre formación inicial del profesorado.

Margarita Pino-Juste, Doctora en Filosofía y Ciencias de la Educación por la Universidad de Santiago de Compostela. Profesora Titular del Departamento de Didáctica, Organización escolar y Métodos de Investigación. Facultad de Ciencias de la Educación y del Deporte. Pontevedra Sus líneas de investigación están centradas en el diseño y desarrollo de programas de educación para la salud y proyectos colaborativos en centros. Ha participado en varios congresos, así como en libros y revistas de impacto nacional e internacional.

Dirección postal de las autoras: Margarita Pino-Juste

Universidad de Vigo

Profesora Titular del Departamento de Didáctica, Organización escolar y Métodos de Investigación.

Facultad de Ciencias de la Educación y del Deporte

Pontevedra

E-mail: mpino@uvigo.es

Margarita González Peiteado UNED

E-mail: margonzalez@pontevedra. uned.es 
Fecha Recepción del Artículo: 15. Diciembre. 2011

Fecha Revisión del Artículo: 22. Abril. 2012

Fecha Aceptación del Artículo: 16. Agosto. 2012

Fecha de Revisión para publicación: 24. Junio. 2013 
